\title{
Changes in High-Density Lipoprotein Cholesterol and Risks of Cardiovascular Events: A Post Hoc Analysis from the PICASSO Trial
}

\author{
Eun-Jae Lee, ${ }^{\mathrm{a}}$ Sun U. Kwon, ${ }^{\mathrm{a}}$ Jong-Ho Park, ${ }^{\mathrm{b}}$ Yong-Jae Kim, ${ }^{\mathrm{c}}$ Keun-Sik Hong, ${ }^{\mathrm{d}}$ Sungwook Yu, ${ }^{\mathrm{e}}$ \\ Yang-Ha Hwang, , Ji Sung Lee, ${ }^{\mathrm{g}}$ Juneyoung Lee, ${ }^{\mathrm{h}}$ Joung-Ho Rha, ${ }^{\mathrm{i}}$ Sung Hyuk Heo, ${ }^{\mathrm{j}}$ Sung Hwan Ahn, ${ }^{\mathrm{k}}$ \\ Woo-Keun Seo, ${ }^{1}$ Jong-Moo Park, ${ }^{\mathrm{m}}$ Ju-Hun Lee, ${ }^{\mathrm{n}}$ Jee-Hyun Kwon, ${ }^{\mathrm{o}}$ Sung-Il Sohn, ${ }^{\mathrm{p}}$ Jin-Man Jung, ${ }^{\mathrm{q}}$ \\ Hahn Young Kim, ${ }^{\mathrm{r}}$ Eung-Gyu Kim, ${ }^{\mathrm{s}}$ Sung Hun Kim, ${ }^{\mathrm{t}}$ Jae-Kwan Cha, ${ }^{\mathrm{u}}$ Man-Seok Park, ${ }^{\mathrm{v}}$ Hyo Suk Nam, ${ }^{\mathrm{w}}$ \\ Dong-Wha Kang, on Behalf of PICASSO Investigators
}

\footnotetext{
${ }^{a}$ Department of Neurology, Asan Medical Center, University of Ulsan College of Medicine, Seoul, Korea Department of Neurology, Myongji Hospital, Hanyang University College of Medicine, Goyang, Korea 'Department of Neurology, Eunpyeong St. Mary's Hospital, College of Medicine, The Catholic University of Korea, Seoul, Korea dDepartment of Neurology, Inje University Ilsan Paik Hospital, Inje University College of Medicine, Goyang, Korea e Department of Neurology, Korea University Anam Hospital, Korea University College of Medicine, Seoul, Korea Department of Neurology, Kyungpook National University Hospital, School of Medicine, Kyungpook National University, Daegu, Korea ${ }^{9}$ Clinical Research Center, Asan Medical Center, University of Ulsan College of Medicine, Seoul, Korea

hepartment of Biostatistics, Korea University, Seoul, Korea

Department of Neurology, Inha University Hospital, Incheon, Korea

Jepartment of Neurology, Kyung Hee University Medical Center, Seoul, Korea

'Department of Neurology, Chosun University Hospital, Chosun University College of Medicine, Gwangju, Korea

Department of Neurology, Samsung Medical Center, Sungkyunkwan University School of Medicine, Seoul, Korea

mDepartment of Neurology, Eulji General Hospital, Eulji University School of Medicine, Seoul, Korea

nepartment of Neurology, Hallym University Kangdong Sacred Heart Hospital, Hallym University College of Medicine, Seoul, Korea

'Department of Neurology, Ulsan University Hospital, University of Ulsan College of Medicine, Ulsan, Korea

pepartment of Neurology, Keimyung University Dongsan Medical Center, Keimyung University School of Medicine, Daegu, Korea

'Department of Neurology, Korea University Ansan Hospital, Korea University College of Medicine, Ansan, Korea

'Department of Neurology, Konkuk University School of Medicine, Seoul, Korea

s. Department of Neurology, Inje University Busan Paik Hospital, Inje University College of Medicine, Busan, Korea

tDepartment of Neurology, Kangwon National University Hospital, Kangwon National University School of Medicine, Chuncheon, Korea

"Department of Neurology, Dong-A University Hospital, Dong-A University College of Medicine, Busan, Korea

Department of Neurology, Chonnam National University Medical School, Gwangju, Korea

wepartment of Neurology, Severance Hospital, Yonsei University College of Medicine, Seoul, Korea
}

Background and purpose Whether pharmacologically altered high-density lipoprotein cholesterol (HDL-C) affects the risk of cardiovascular events is unknown. Recently, we have reported the Prevention of Cardiovascular Events in Asian Patients with Ischaemic Stroke at High Risk of Cerebral Haemorrhage (PICASSO) trial that demonstrated the non-inferiority of cilostazol to aspirin and superiority of probucol to non-probucol for cardiovascular prevention in ischemic stroke patients (clinicaltrials.gov: NCT01013532). We aimed to determine whether on-treatment HDL-C changes by cilostazol and probucol influence the treatment effect of each study medication during the PICASSO study.
Correspondence: Sun U. Kwon Department of Neurology, Asan Medical Center, University of Ulsan College of Medicine, 88 Olympic-ro 43-gil, Songpa-gu, Seoul 05505, Korea Tel: +82-2-3010-3960 Fax: +82-2-474-4691 E-mail: sukwon@amc.seoul.kr

Received: September 23, 2019 Revised: November 30, 2019 Accepted: December 11, 2019 
Methods Of the 1,534 randomized patients, 1,373 (89.5\%) with baseline cholesterol parameters were analyzed. Efficacy endpoint was the composite of stroke, myocardial infarction, and cardiovascular death. Cox proportional hazards regression analysis examined an interaction between the treatment effect and changes in HDL-C levels from randomization to 1 month for each study arm.

Results One-month post-randomization mean HDL-C level was significantly higher in the cilostazol group than in the aspirin group $(1.08 \mathrm{mmol} / \mathrm{L}$ vs. $1.00 \mathrm{mmol} / \mathrm{L}, P<0.001)$. The mean HDL-C level was significantly lower in the probucol group than in the non-probucol group $(0.86 \mathrm{mmol} / \mathrm{L}$ vs. 1.22 $\mathrm{mmol} / \mathrm{L}, P<0.001)$. These trends persisted throughout the study. In both study arms, no significant interaction was observed between $\mathrm{HDL}-\mathrm{C}$ changes and the assigned treatment regarding the risk of the efficacy endpoint.

Conclusions Despite significant HDL-C changes, the effects of cilostazol and probucol treatment on the risk of cardiovascular events were insignificant. Pharmacologically altered HDL-C levels may not be reliable prognostic markers for cardiovascular risk.

Keywords Cholesterol, HDL; Secondary prevention; Cilostazol; Probucol; Cholesterol ester transfer proteins

\section{Introduction}

High-density lipoprotein cholesterol (HDL-C) has shown an inverse association between its level and the risk of cardiovascular disease in observational studies..$^{1-3}$ This association has also been persistent in most post hoc analyses and meta-analyses of statin trials. ${ }^{4-8} \mathrm{HDLs}$ contribute to the process of cellular cholesterol efflux; therefore, pharmacological elevation of HDL-C levels may improve cardiovascular outcomes. However, recent clinical trials testing the efficacy of cholesteryl ester transfer protein (CETP) inhibitors that increase HDL-C levels have failed to demonstrate definite clinical benefits ${ }^{9-11}$ due to a lack of significant association between HDL-C levels per se and cardiovascular risk; however, the intrinsic nature of CETP inhibitors (e.g., increasing atherogenic apoproteins) may also have affected the results. ${ }^{12}$ Pharmacologically altered HDL-C levels having different mechanisms may give rise to different results.

HDL-C can be altered by medications other than CETP inhibitors during secondary cardiovascular prevention. For instance, cilostazol has been reported to increase HDL-C levels by activating lipoprotein lipase. ${ }^{13-16}$ Meanwhile, probucol upregulates CETP that significantly decreases HDL-C levels, ${ }^{17}$ which has been considered a deleterious side effect, preventing the widespread use of probucol. ${ }^{17,18}$

In this study, we hypothesized that medications altering HDL-C levels may influence cardiovascular risks. To test this hypothesis, we used the recent published data of the Prevention of Cardiovascular Events in Asian Patients with Ischaemic Stroke at High Risk of Cerebral Haemorrhage (PICASSO) study..$^{19}$ In the study, cilostazol was non-inferior to aspirin for the pre- vention of cardiovascular events, while the addition of probucol to aspirin or cilostazol was superior to non-probucol treatment. Notably, the opposite study medications (cilostazol and probucol) in terms of HDL-C alteration were administered in the study. Here, we aimed to determine whether on-treatment changes in HDL-C levels induced by cilostazol and probucol would influence the treatment effect of each study medication.

\section{Methods}

\section{Study design and population}

The PICASSO trial had a factorial design consisting of two main study arms-antiplatelet regimens (cilostazol vs. aspirin) and lipid-lowering regimens (standard statin-based therapy plus probucol vs. standard statin-based therapy only). The rationale, design, and relevant information of the study have been previously described. ${ }^{19,20}$

Briefly, we included patients who (1) had a history of a noncardioembolic ischemic stroke or transient ischemic attack within 180 days prior to enrollment; (2) were older than 20 years; and (3) had a history of either a previous intracerebral hemorrhage (ICH) based on clinical history or radiologic findings (over $8 \mathrm{~mm}$ in size on gradient echo imaging) or multiple (two or more) cerebral microbleeds on gradient echo imaging. We excluded patients who (1) had a history of a hemorrhagic stroke within the past 6 months; (2) had conditions contraindicating long-term antiplatelet therapy; and (3) required dual antiplatelet therapy for a recent acute coronary syndrome or a percutaneous coronary intervention. Participants who met the criteria were consecutively recruited by local investigators. All participants or 
their legally authorized representatives provided informed consent prior to study enrollment.

Between August 2009 and August 2015, 1,568 patients who recovered from stroke from 67 centers were initially screened in three countries (South Korea, China [Hong Kong], and Philippines), and 1,534 were enrolled in the study (Figure 1). Patients were randomly assigned (1:1:1:1) to receive oral cilostazol (100 $\mathrm{mg}$ twice a day), aspirin (100 mg once a day), cilostazol plus probucol (250 mg twice a day), or aspirin plus probucol. Adherence to statin therapy as outlined in clinical practice guidelines was strongly recommended. The antiplatelet arm was a doubleblind, double-dummy, placebo-controlled, randomized trial, while the probucol arm was an open-labeled, blind endpoint evaluation trial. The outcome assessor was blinded to the participants' treatment assignment.

The trial protocol was conducted in accordance with the Good Clinical Practice Guidelines and the Declaration of Helsinki and was approved by the respective ethics committees and Institutional Review Boards of all participating local centers. This study was registered at the ClinicalTrials.gov (NCT01013532).

\section{Follow-up and outcomes}

Patients' follow-up visits took place at 1, 4, 7, 10, and 13 months after randomization; thereafter, patients visited the centers annually. Lipid levels (low-density lipoprotein cholesterol [LDL-C], HDL-C, and triglycerides) were assessed at baseline (i.e., at randomization), at 1-month follow-up visit, and annually thereafter. Blood test for cholesterol profile was rec- ommended in all patients; however, patients who refused to undergo the test were still included in the PICASSO trial.

The primary efficacy endpoint was a composite of stroke, myocardial infarction, and cardiovascular death. The primary safety endpoint was hemorrhagic stroke, including spontaneous ICH and subarachnoid hemorrhage. All reported endpoints of interest were blindly judged by the Central Independent Adjudication Committee at the regular meeting.

\section{Statistical analysis}

Patients' baseline characteristics were compared between the treatment groups with Pearson's chi-square tests or Fisher's exact test for categorical variables and t-test or Wilcoxon ranksum test for continuous variables, as appropriate. Longitudinal changes in cholesterol levels (LDL-C, HDL-C, and triglycerides) were compared between the study groups using the generalized estimating equations method. To examine whether the non-inferiority of cilostazol to aspirin and superiority of probucol to non-probucol were evident in our study population as in the previous study, ${ }_{1}^{19}$ we first tested the efficacy of the study medications to prevent the composite primary efficacy endpoint; non-inferiority was observed if the upper limit of the 95\% confidence intervals (Cls) of the hazard ratio (HR) did not exceed 1.25. Additionally, Cox proportional hazards models were used to calculate HRs for the outcome variables.

We evaluated whether the treatment effect of the study medication was influenced by HDL-C alterations during the study. Poisson regression was performed according to HDL-C changes from baseline to on-treatment levels measured 1 month after

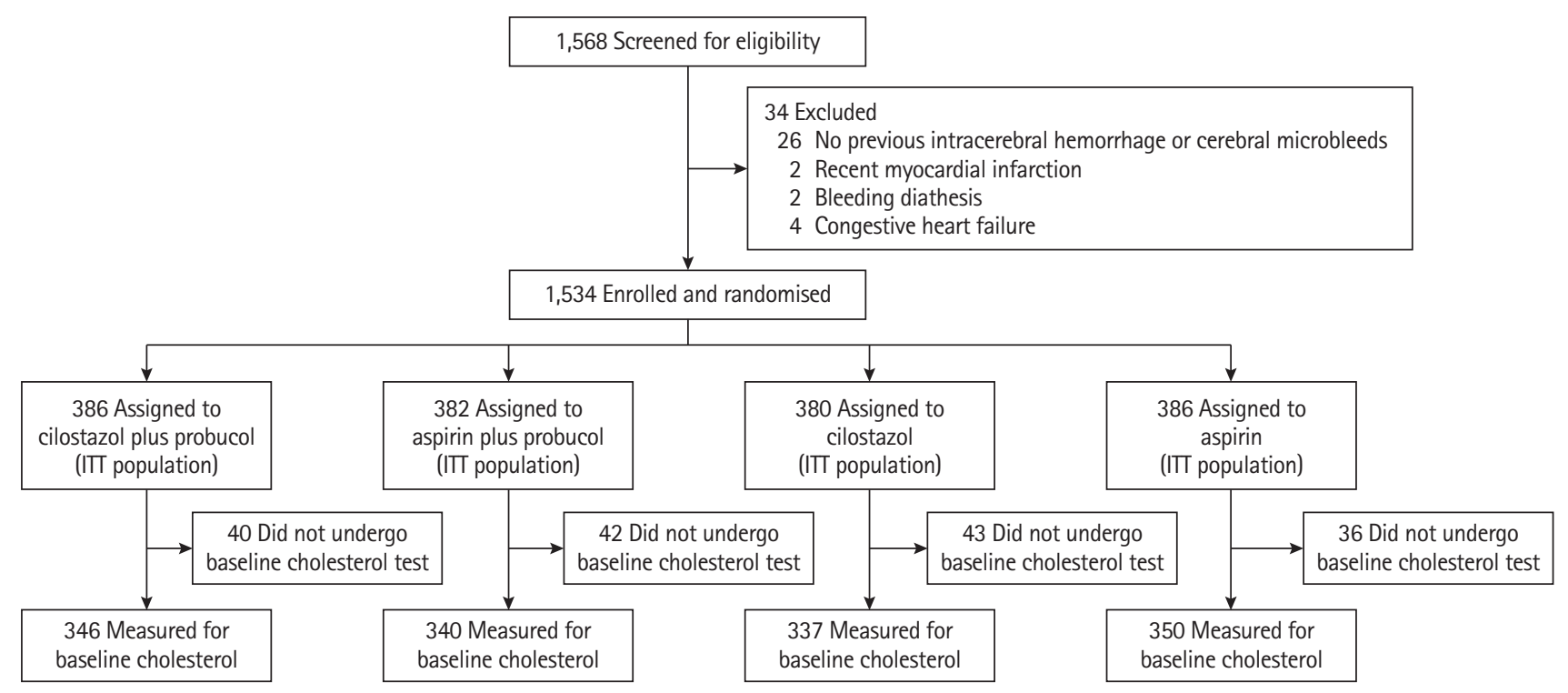

Figure 1. Trial profile. Among the intention-to-treat (ITT) population including all randomized patients in the Prevention of Cardiovascular Events in Asian Patients with Ischaemic Stroke at High Risk of Cerebral Haemorrhage study, those who underwent baseline cholesterol evaluation were analyzed in this study. 
randomization; quartile categories for HDL-C changes were separately obtained for each treatment arm. In this analysis, the outcome variables 1 month after randomization were evaluated. Considering that there was no outcome event that had developed during this initial 1-month period, any patient with early outcomes in this post hoc analysis was not excluded. A complete case analysis was performed using only cases with no missing values. The annualized event rates (\%) and $95 \% \mathrm{Cls}$ in the quartile groups were estimated and compared. Finally, Cox proportional hazards regression analysis was performed to examine an interaction effect between the treatment group and changes in HDL-C levels for 1 month. To compare the treatment groups, we used a two-sided significance level of 0.05 . All statistical analyses were performed using the Statistical Analysis System (SAS) version 9.4 (SAS Institute Inc., Cary, NC, USA).

\section{Results}

Of the 1,534 patients randomized in the PICASSO trial, 1,373 (89.5\%) with baseline cholesterol parameters were analyzed in this study (Figure 1). The median age was $66.1 \pm 10.8$ years, and a total of 530 participants (38.6\%) were female. The median duration of follow-up was 1.9 years (interquartile range, 1.0 to 3.0). Considering the $2 \times 2$ factorial design of the PICASSO trial, the interaction between antiplatelet therapy and probucol therapy was assessed before the effect of each treatment arm was evaluated. Significant interactions were not observed:19 thus, the effects on outcomes of antiplatelet drugs and probucol were separately analyzed. Study groups' demographic and clinical characteristics were well balanced (Table 1). Statin treatment was initiated in 1,080 patients $(78.7 \%)$ at baseline.

The cholesterol profiles over time are shown in Figure 2. In the antiplatelet study arm, cilostazol users showed significantly higher HDL-C levels than aspirin users at 1 month (mean HDL-C, cilostazol vs. aspirin, $41.8 \mathrm{mg} / \mathrm{dL}$ [1.08 mmol/L] vs. $38.8 \mathrm{mg} / \mathrm{dL}$ $[1.00 \mathrm{mmol} / \mathrm{L}]$ ). This trend persisted throughout the study (Figure 2A). Additionally, triglyceride levels were significantly lower at 1 month with cilostazol treatment than with aspirin treatment (mean triglyceride, cilostazol vs. aspirin, $100.8 \mathrm{mg} / \mathrm{dL}$ [1.14 $\mathrm{mmol} / \mathrm{L}]$ vs. $124.9 \mathrm{mg} / \mathrm{dL}[1.41 \mathrm{mmol} / \mathrm{L}])$, and this trend was also maintained thereafter (Figure 2B). Regarding LDL-C, both treatment groups showed comparable levels during the study, except at 13 months after treatment (Figure $2 \mathrm{C}$ ).

In the probucol study arm, HDL-C levels significantly decreased in the probucol group but increased in the non-probucol group 1 month after randomization. The difference between the treatment groups was significant (mean HDL-C at 1 month, probucol vs. non-probucol, $33.4 \mathrm{mg} / \mathrm{dL}$ [0.86 $\mathrm{mmol} / \mathrm{L}]$ vs. 47.3 $\mathrm{mg} / \mathrm{dL}$ [1.22 $\mathrm{mmol} / \mathrm{L}])$ (Figure 2D). Triglyceride levels were also affected by probucol treatment at 1 and 13 months after treatment, but the difference was not as significant as in the antiplatelet study arm (Figure 2E). LDL-C levels were significantly lower in the probucol group than in the non-probucol group 1 month after randomization (mean LDL-C at 1 month, probucol vs. non-probucol, $74.2 \mathrm{mg} / \mathrm{dL}$ [1.92 $\mathrm{mmol} / \mathrm{L}]$ vs. 78.4 $\mathrm{mg} / \mathrm{dL}$ [2.23 mmol/dL]) (Figure 2F).

Outcome analyses are shown in Table 2. Consistent with the intention-to-treat analysis, ${ }^{19}$ cilostazol treatment was non-inferior to aspirin $(P=0.006)$ but not superior to aspirin in a superiority test $(P=0.137)$ in the prevention of the primary efficacy outcomes. The safety outcome, hemorrhagic stroke, tended to occur less frequently in the cilostazol group than in the other groups, but this difference was statistically insignificant. In the probucol arm, addition of probucol to cilostazol or aspirin was non-inferior $(P=0.001)$ and superior $(P=0.033)$ to cilostazol or aspirin alone. Probucol treatment did not increase the risk of the safety outcome.

Subsequently, we analyzed whether increased and decreased HDL-C levels altered the annualized event rates of the primary efficacy endpoint in the study medication groups (Figure 3). At 1 month after randomization, the distribution of HDL-C levels had shifted rightward as a result of cilostazol treatment, while it had shifted leftward with probucol treatment. The annualized risk of the endpoints 1 month after randomization was evaluated according to quartiles of change in HDL-C levels from baseline to 1 month. As a result, there was no significant main effect of the change in HDL-C levels regarding the risk of the primary endpoint within each treatment group $(P=0.508$ in the cilostazol group, $P=0.894$ in the aspirin group, $P=0.841$ in the probucol group, $P=0.779$ in the non-probucol group). Moreover, there was also no significant interaction between treatment group allocation and HDL-C changes in terms of the risk of the primary efficacy endpoint ( $P=0.623$ in the antiplatelet arm, $P=0.997$ in the probucol arm) (Table 3). Notably, the annualized event rate of the primary efficacy endpoint in the probucol group was consistently lower than that in the non-probucol group throughout all quartiles of HDL-C changes (Figure $3 \mathrm{C}$ ).

Finally, we performed Cox proportional hazards regression analysis to evaluate an interaction effect between the treatment effects of each medication (cilostazol vs. aspirin; probucol vs. non-probucol) and numerical changes in HDL-C levels from baseline to 1 month. No significant interactions between the treatment effect (HRs for the efficacy endpoint) and HDL-C alterations were observed in both study arms $(P=0.158$ for cilostazol in the antiplatelet arm, $P=0.532$ for probucol in the probucol arm) (Figure 4). 
Table 1. Baseline characteristics

\begin{tabular}{|c|c|c|c|c|c|c|}
\hline \multirow[b]{2}{*}{ Characteristic } & \multicolumn{3}{|c|}{ Antiplatelet arm } & \multicolumn{3}{|c|}{ Probucol arm } \\
\hline & $\begin{array}{l}\text { Cilostazol } \\
(n=683)\end{array}$ & $\begin{array}{l}\text { Aspirin } \\
(n=690)\end{array}$ & $P$ & $\begin{array}{l}\text { Probucol } \\
(n=686)\end{array}$ & $\begin{array}{l}\text { Non-probucol } \\
\quad(n=687)\end{array}$ & $P$ \\
\hline Age (yr) & $65.9 \pm 10.8$ & $66.2 \pm 10.7$ & 0.601 & $65.8 \pm 10.8$ & $66.3 \pm 10.7$ & 0.379 \\
\hline Male sex & $421(62)$ & $422(61)$ & 0.855 & $417(61)$ & $426(62)$ & 0.642 \\
\hline Body mass index $\left(\mathrm{kg} / \mathrm{m}^{2}\right)$ & $24.4 \pm 3.5$ & $24.3 \pm 3.5$ & 0.479 & $24.5 \pm 3.5$ & $24.2 \pm 3.5$ & 0.087 \\
\hline Entry event & & & 0.638 & & & 0.126 \\
\hline Ischemic stroke & $653(96)$ & 656 (95) & & $660(96)$ & 649 (94) & \\
\hline Transient ischemic attack & $30(4)$ & $34(5)$ & & $26(4)$ & $38(6)$ & \\
\hline Index of high risk of ICH & & & 0.907 & & & 0.791 \\
\hline Prior history of $\mathrm{ICH}$ & $140(20)$ & $144(21)$ & & $137(20)$ & $147(21)$ & \\
\hline $\begin{array}{l}\text { Imaging findings of ICH without clinical } \\
\text { history }\end{array}$ & $129(19)$ & 124 (18) & & $129(19)$ & 124 (18) & \\
\hline Multiple microbleeds & $414(61)$ & $422(61)$ & & $420(61)$ & $416(61)$ & \\
\hline $\begin{array}{l}\text { Time-to-randomization since entry event } \\
\text { (day) }\end{array}$ & $18(8-40)$ & $17(8-36)$ & 0.615 & $18(8-38)$ & $17(8-38)$ & 0.417 \\
\hline \multicolumn{7}{|l|}{ Risk factors } \\
\hline Hypertension & $603(88)$ & 614 (89) & 0.683 & $614(90)$ & 603 (88) & 0.312 \\
\hline Diabetes & 214 (31) & 226 (33) & 0.573 & $211(31)$ & 229 (33) & 0.307 \\
\hline Current smoking & $135(20)$ & $149(22)$ & 0.403 & $144(21)$ & $140(20)$ & 0.779 \\
\hline \multicolumn{7}{|l|}{ Lipids (mg/dL)* } \\
\hline $\mathrm{HDL}-\mathrm{C}$ & $45.1 \pm 11.8$ & $45.3 \pm 12.2$ & 0.847 & $45.3 \pm 12.1$ & $45.1 \pm 11.9$ & 0.689 \\
\hline LDL-C & $102.3 \pm 36.7$ & $102.9 \pm 34.8$ & 0.748 & $104.5 \pm 35.8$ & $100.7 \pm 35.6$ & 0.047 \\
\hline Triglycerides & $125.3 \pm 89.4$ & $132.6 \pm 83.2$ & 0.116 & $131.9 \pm 93.6$ & $126.1 \pm 78.6$ & 0.218 \\
\hline Fazekas score $^{\dagger}$ & & & 0.505 & & & 0.440 \\
\hline 0 & $0(0)$ & $2(0)$ & & $1(0)$ & $1(0)$ & \\
\hline 1 & $184(28)$ & $203(30)$ & & $186(28)$ & $201(31)$ & \\
\hline 2 & $296(45)$ & $290(43)$ & & $309(46)$ & $277(42)$ & \\
\hline 3 & $174(27)$ & $176(26)$ & & $171(26)$ & $179(27)$ & \\
\hline Intima-media thickness & $1.11 \pm 0.13$ & $1.10 \pm 0.13$ & 0.229 & $1.11 \pm 0.13$ & $1.10 \pm 0.13$ & 0.113 \\
\hline Ankle-brachial index & $0.91 \pm 0.25$ & $0.93 \pm 0.24$ & 0.353 & $0.92 \pm 0.25$ & $0.92 \pm 0.24$ & 0.897 \\
\hline Concomitant therapy & & & & & & 0.608 \\
\hline Statin & $535(78)$ & $545(79)$ & 0.767 & $539(79)$ & $541(79)$ & 0.936 \\
\hline Low intensity & $8(2)$ & $9(2)$ & & $8(2)$ & $9(2)$ & \\
\hline Moderate intensity & $313(61)$ & 309 (59) & 0.848 & 317 (62) & 305 (59) & 0.655 \\
\hline High intensity & $191(37)$ & 202 (39) & & $189(37)$ & 204 (39) & \\
\hline ARB/ACEI & $414(61)$ & $372(54)$ & 0.012 & 391 (57) & 395 (57) & 0.852 \\
\hline Dihydropyridine & $323(47)$ & $315(46)$ & 0.543 & $315(46)$ & $323(47)$ & 0.683 \\
\hline Beta blockers & $72(11)$ & $70(10)$ & 0.809 & 77 (11) & $65(9)$ & 0.283 \\
\hline Thiazide & $98(14)$ & $86(12)$ & 0.305 & $97(14)$ & 87 (13) & 0.422 \\
\hline
\end{tabular}

Values are presented as mean \pm standard deviation, number (\%), or median (interquartile range).

ICH, intracerebral hemorrhage; HDL-C, high-density lipoprotein cholesterol; LDL-C, low-density lipoprotein cholesterol; ARB, angiotensin II receptor blocker; ACEl, angiotensin-converting enzyme inhibitor.

${ }^{*}$ To convert cholesterol and triglyceride values to millimoles per liter, multiply by 0.02586 and 0.01129 , respectively; ${ }^{+}$Data evaluated for patients who underwent magnetic resonance imaging with fluid-attenuated inversion recovery sequences. 

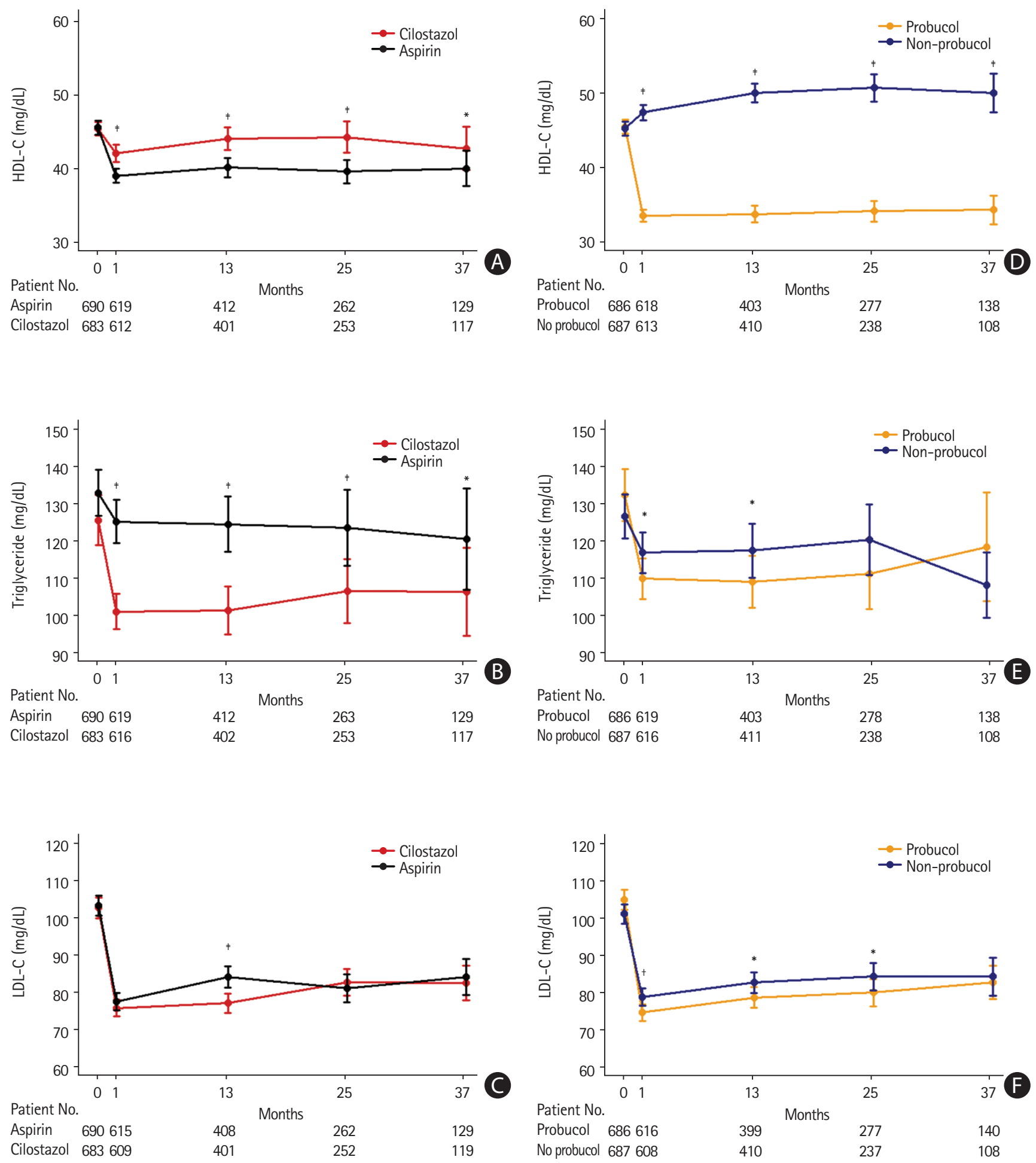

Figure 2. Cholesterol profiles over time. (A) High-density lipoprotein cholesterol (HDL-C), (B) triglyceride, and (C) low-density lipoprotein cholesterol (LDL-C) levels in the antiplatelet study arm, (D) HDL-C, (E) triglyceride, and (F) LDL-C levels in the probucol study arm. The $P$-values using the generalized estimated equations for probucol-by-visit interaction: cilostazol vs. aspirin, $\mathrm{HDL}-\mathrm{C}(P<0.001)$, triglycerides $(P=0.004), \mathrm{LDL}-\mathrm{C}(P=0.007)$; probucol vs. non-probucol, HDL-C $(P<0.001)$, triglycerides $(P=0.003)$, LDL-C $(P=0.004) .{ }^{*} P<0.05 ;{ }^{\dagger} P<0.01 ;{ }^{*} P<0.001$. 
Table 2. Primary outcome measures in this study population

\begin{tabular}{|c|c|c|c|c|c|c|c|c|}
\hline \multirow[b]{2}{*}{ Variable } & \multicolumn{4}{|c|}{ Antiplatelet arm } & \multicolumn{4}{|c|}{ Probucol arm } \\
\hline & $\begin{array}{c}\text { Cilostazol } \\
(n=683)\end{array}$ & $\begin{array}{l}\text { Aspirin } \\
(n=690)\end{array}$ & HR (95\% Cl) & $P$ & $\begin{array}{l}\text { Probucol } \\
(n=686)\end{array}$ & $\begin{array}{c}\text { Non-probucol } \\
(n=687)\end{array}$ & $\mathrm{HR}(95 \% \mathrm{Cl})$ & $P$ \\
\hline \multicolumn{9}{|l|}{ Efficacy endpoint* } \\
\hline Composite vascular events & & & $0.77(0.54-1.09)$ & $\begin{array}{l}0.006^{\dagger} \\
0.137^{\ddagger}\end{array}$ & & & $0.68(0.48-0.97)$ & $\begin{array}{l}0.001^{\dagger} \\
0.033^{\ddagger}\end{array}$ \\
\hline No. of events & 55 & 73 & & & 53 & 75 & & \\
\hline Rate (\%/patient-year) & $4.06(3.11-5.28)$ & $5.27(4.19-6.62)$ & & & $3.76(2.87-4.92)$ & $5.63(4.49-7.07)$ & & \\
\hline \multicolumn{9}{|l|}{ Safety endpoint ${ }^{\S}$} \\
\hline Hemorrhagic stroke & & & $0.42(0.17-1.02)$ & 0.054 & & & $0.68(0.30-1.54)$ & 0.359 \\
\hline No. of events & 7 & 17 & & & 10 & 14 & & \\
\hline Rate (\%/patient-year) & $0.52(0.25-1.08)$ & $1.22(0.76-1.97)$ & & & $0.71(0.38-1.32)$ & $1.05(0.62-1.77)$ & & \\
\hline
\end{tabular}

$\mathrm{HR}$, hazard ratio; $\mathrm{Cl}$, confidence interval.

*Efficacy endpoint: a composite of stroke, myocardial infarction, and cardiovascular death; ${ }^{\dagger} P$-value for non-inferiority test with a non-inferiority margin of

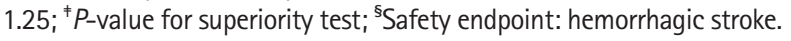

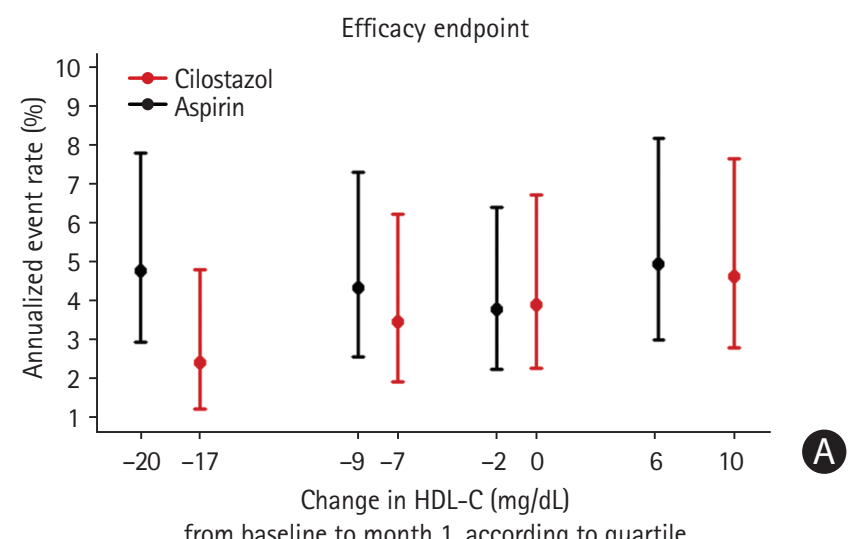

from baseline to month 1 , according to quartile

Efficacy endpoint

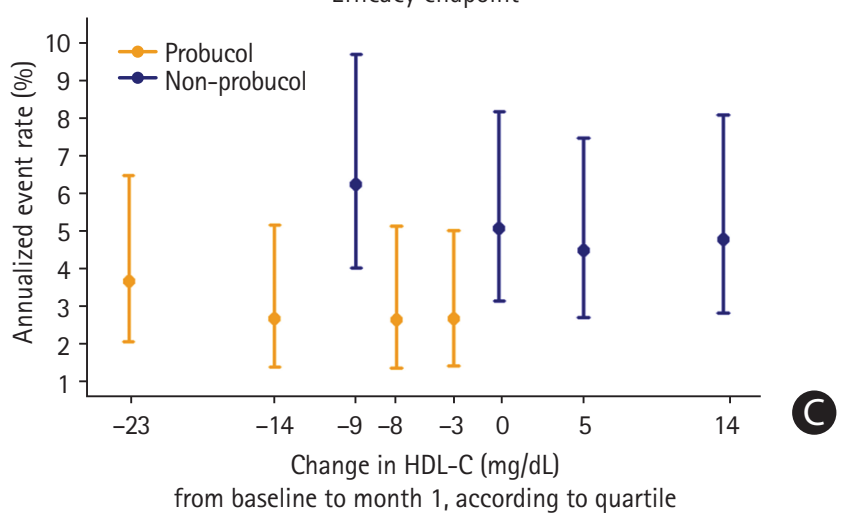

from baseline to month 1 , according to quartile
Safety endpoint

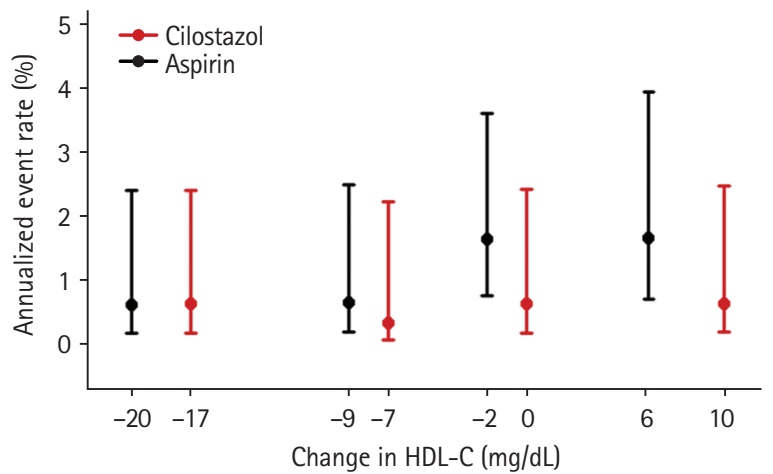

from baseline to month 1 , according to quartile

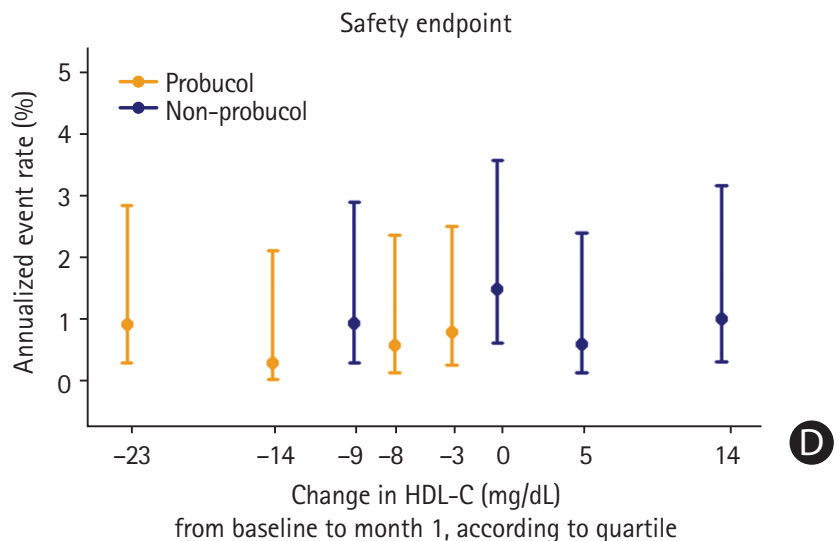

Figure 3. The annualized risk of the primary endpoint beginning at 1 month, according to quartiles of change in high-density lipoprotein cholesterol (HDL-C) levels from baseline to 1 month after randomization. (A) Efficacy endpoint (a composite of stroke, myocardial infarction, and cardiovascular death), (B) safety endpoint (cerebral hemorrhage) in the antiplatelet study arm, (C) efficacy endpoint, and (D) safety endpoint in the probucol study arm. The position of each quartile of HDL-C on the $\mathrm{x}$-axis represents the median value of HDL-C changes within that quartile. 
Table 3. Annual event rate according to quartiles of high-density lipoprotein cholesterol 1 month after randomization

\begin{tabular}{|c|c|c|c|c|c|c|c|c|}
\hline \multirow{2}{*}{ Variable } & \multicolumn{4}{|c|}{ Antiplatelet arm } & \multicolumn{4}{|c|}{ Probucol arm } \\
\hline & Cilostazol & Aspirin & $P^{*}$ & $P^{+}$ & Probucol & Non-probucol & $P^{*}$ & $P^{+}$ \\
\hline Efficacy endpoint ${ }^{+}$ & & & 0.623 & & & & 0.997 & \\
\hline 1st quartile & $2.40(1.20-4.79)$ & $4.77(2.92-7.79)$ & & 0.112 & $3.69(2.09-6.50)$ & $6.25(4.03-9.69)$ & & 0.149 \\
\hline 2nd quartile & $3.44(1.90-6.21)$ & $4.33(2.56-7.31)$ & & 0.568 & $2.70(1.40-5.18)$ & $5.09(3.16-8.18)$ & & 0.123 \\
\hline 3rd quartile & $3.90(2.27-6.72)$ & $3.79(2.24-6.39)$ & & 0.937 & $2.68(1.39-5.15)$ & $4.51(2.72-7.47)$ & & 0.217 \\
\hline 4th quartile & $4.61(2.78-7.64)$ & $4.93(2.97-8.18)$ & & 0.852 & $2.70(1.45-5.02)$ & $4.79(2.84-8.08)$ & & 0.166 \\
\hline Safety endpoint ${ }^{\S}$ & & & 0.864 & & & & 0.655 & \\
\hline 1st quartile & $0.60(0.15-2.40)$ & $0.60(0.15-2.38)$ & & 0.996 & $0.92(0.30-2.86)$ & $0.94(0.30-2.91)$ & & 0.984 \\
\hline 2nd quartile & $0.31(0.04-2.22)$ & $0.62(0.15-2.47)$ & & 0.578 & $0.30(0.04-2.13)$ & $1.49(0.62-3.59)$ & & 0.142 \\
\hline 3rd quartile & $0.60(0.15-2.40)$ & $1.62(0.73-3.61)$ & & 0.224 & $0.60(0.15-2.38)$ & $0.60(0.15-2.40)$ & & 0.994 \\
\hline 4th quartile & $0.61(0.15-2.45)$ & $1.64(0.68-3.95)$ & & 0.239 & $0.81(0.26-2.51)$ & $1.03(0.33-3.18)$ & & 0.772 \\
\hline
\end{tabular}

Values are presented as annualized event rate (\%) and $95 \%$ confidence interval.

${ }^{*} P$-value by Poisson regression for treatment-by-quartile interaction; ${ }^{+} P$-value by contrast test with Poisson regression for each quartile; ${ }^{*}$ Efficacy endpoint: a composite of stroke, myocardial infarction, and cardiovascular death; 'Safety endpoint: hemorrhagic stroke.
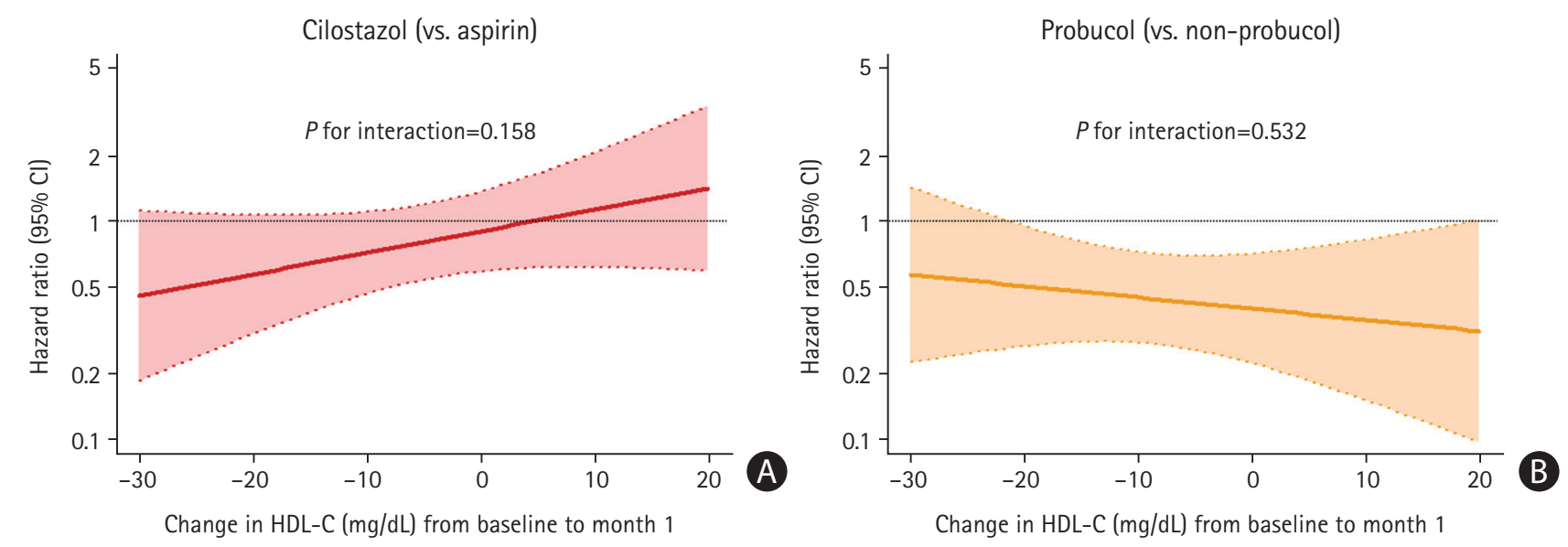

Figure 4. Hazard ratio for the composite vascular event of the study medication group vs. the control group according to numerical changes in high-density lipoprotein cholesterol (HDL-C) levels from baseline to 1-month post-randomization. (A) Cilostazol vs. aspirin, (B) probucol vs. non-probucol. No significant interactions between the treatment effect and $\mathrm{HDL}-\mathrm{C}$ alterations were observed in both study arms (Cox proportional hazards regression analysis, $P=0.158$ for cilostazol in the antiplatelet arm, $P=0.532$ for probucol in the probucol arm). $\mathrm{Cl}$, confidence interval.

\section{Discussion}

We investigated whether the efficacy of study medications was altered with on-treatment HDL-C changes in patients with an ischemic stroke at high risk of cerebral hemorrhage. Cilostazol and probucol treatment demonstrated opposite effects on HDL-C levels during the study; however, both changes were insignificant. These findings suggest that pharmacologically altered HDL-C levels and their changes during active prevention may not be reliable prognostic markers to estimate the risks of cardiovascular events.
Regarding cholesterol profiles, patients who received cilostazol showed higher HDL-C levels and lower triglyceride levels than those who received aspirin. These changes are consistent with that of the previous reports ${ }^{13-16}$ and may be attributable to increased lipoprotein lipase activity. ${ }^{14}$ In the probucol study arm, both HDL-C and LDL-C levels were remarkably lower in the probucol group than in the non-probucol group, while triglycerides were relatively comparable between the treatment groups. These profiles were also consistent with the previous study findings. ${ }^{21-23}$ The upregulation of CETP by probucol was associated with increased HDL-C levels. ${ }^{17}$ 
Regarding the outcome analyses, non-inferiority of cilostazol to aspirin treatment in reducing composite vascular outcomes was evident in our population. Importantly, the post hoc analyses of endpoints did not show any significant interaction between the cilostazol effect and HDL-C changes (Figures 3 and 4), suggesting that increased HDL-C levels may not enhance the drug efficacy in secondary prevention. Meanwhile, probucol treatment was superior to non-probucol treatment in preventing the primary efficacy endpoint in this study population. The treatment effects of probucol were also comparable, regardless of HDL-C changes (Figures 3 and 4), suggesting that the clinical benefits of the drug may not be affected by a decrease in HDL-C levels. Meta-analyses performed in 17 genetic studies revealed that a causal role for HDL-C on the risk of coronary heart disease is not evident. ${ }^{24}$ Additionally, genetic mechanisms that increase HDL-C levels did not lower the risk of myocardial infarction. ${ }^{25}$ Accordingly, recent large clinical trials assessing the efficacy of the CETP inhibitors have not demonstrated definite clinical benefits in patients with high cardiovascular risk. Despite increases in HDL-C levels by greater than $70 \%$ to $100 \%$, torcetrapib and dalcetrapib failed to prove their efficacy in secondary prevention. ${ }^{9,10}$ Anacetrapib showed a modest benefit by reducing major coronary events:;1 however, its effect could not be attributed solely to the fact that it increased HDL-C-levels because it also significantly lowered LDL-C levels. ${ }^{12}$

The following are the several potential explanations regarding the lack of association between HDL-C levels and clinical benefits: ${ }^{10,26}$ (1) HDL-C level may lose its role as a determinant of cardiovascular risk when patients are treated with evidencebased therapy in a clinical trial setting; (2) the protective effect of HDL-C may be limited to healthy individuals, not patients who already have cardiovascular disease; (3) the measured HDL-C levels may not fully reflect the physiologic functions of HDLs; and (4) cholesterol efflux capacity rather than HDL-C level per se is the crucial determinant of cardiovascular risk. ${ }^{26}$ Therefore, probucol's HDL-C-lowering effect does not necessarily have to be considered a significant adverse effect.

What is the mechanism for beneficial effects of probucol? A greater reduction in LDL-C in probucol users may not explain the clinical benefit of probucol because the degree of difference between the treatment groups was less than $5 \mathrm{mg} / \mathrm{dL}(0.1 \mathrm{mmol} / \mathrm{L})$ throughout the study. Considering that a $20 \%$ reduction in the risk of cardiovascular disease is achieved by each reduction of 40 $\mathrm{mg} / \mathrm{dL}(1 \mathrm{mmol} / \mathrm{L})$ in the LDL-C level, ${ }^{5}$ a $31 \%$ reduction in the incidence of the primary endpoint in our study may have come from a decrease in the residual cardiovascular risk rather than from LDL-C lowering activity. The upregulation of CETP may have converted the whole HDL-C into smaller particles that are more effectively excreted ${ }_{1}^{27}$ decreasing the body's total cholesterol burden. Additionally, other known pleiotropic effects of the drug may have induced the observed clinical benefits. ${ }^{17,18,28-30}$ Particularly, endothelial dysfunction and increased vascular permeability are associated with ischemic and hemorrhagic strokes. ${ }^{31}$ These strokes are the clinical characteristics of our participants, and probucol may have had beneficial effects in both of these factors.

This study has several limitations. First is the study's post hoc design, which is prone to be affected by chance and confounding biases. Second, the number of study participants was not determined to examine the prognostic value of HDL-C levels; hence, the study may not have sufficient power to verify the effect of on-treatment HDL-C changes on the risk of cardiovascular events. Therefore, the results of this study should be cautiously interpreted and regarded as an observational evidence. Third, cholesterol profiles are not available in all patients. Despite a profile that is evident, particularly for HDL-C levels, the results of this profile should be cautiously interpreted. Fourth, the degree of HDL-C increases by cilostazol may be significantly small to manifest its effect in enhancing the treatment efficacy of the study medication. Previous trials to test CETP inhibitors have resulted in a greater difference on HDL-C levels between the treatment groups (e.g., mean HDL-C level during the study: dalcetrapib vs. placebo, $55 \mathrm{mg} / \mathrm{dL}$ [1.42 $\mathrm{mmol} / \mathrm{L}]$ vs. $43 \mathrm{mg} / \mathrm{dL}$ [1.11 $\mathrm{mmol} / \mathrm{L}]$ at 1 month; anacetrapib vs. placebo, $85 \mathrm{mg} / \mathrm{dL}$ [2.20 mmol/L] vs. 42 $\mathrm{mg} / \mathrm{dL}[1.09 \mathrm{mmol} / \mathrm{L}]$ at midpoint). ${ }^{10,11}$ However, it should also be noted that these CETP inhibitors have demonstrated only modest or no clinical benefits. Fifth, we did not consider the subtypes of stroke in our analyses, such as Trial of Org 10172 in Acute Stroke Treatment classification, because information about cerebral vessels has not been obtained. This point should be considered because the detrimental effect of HDL-C reduction may be less noticeable in patients with small-vessel occlusion. This occlusion may be a more common stroke subtype in our participants compared to individuals with large artery atherosclerosis. Finally, because only Asian patients are enrolled in this study, the results may not be generalizable to other ethnicities.

\section{Conclusions}

It is evident that cilostazol and probucol treatment caused significant HDL-C changes during the PICASSO study. Notably, cardiovascular risks and their treatment efficacy were not influenced by on-treatment HDL-C changes. These findings suggest that pharmacologically altered HDL-C levels may not be reliable prognostic markers for cardiovascular risk, reaffirming the recent failures of CETP inhibitor trials. 


\section{Disclosure}

Dr. Sun U. Kwon had received grants from Korea Otsuka Pharmaceutical for PICASSO study, not for this analysis. Other authors have no conflict of interest to declare.

\section{Acknowledgments}

Korea Otsuka Pharmaceutical for PICASSO study, Ministry of Health \& Welfare (HI18C2383) and Ministry of Science and ICT (NRF-2018M3A9E8066249), Republic of Korea.

\section{References}

1. Assmann $G$, Schulte $H$. Relation of high-density lipoprotein cholesterol and triglycerides to incidence of atherosclerotic coronary artery disease (the PROCAM experience). Prospective Cardiovascular Münster study. Am J Cardiol 1992;70:733-737.

2. Gordon T, Castelli WP, Hjortland MC, Kannel WB, Dawber TR. High density lipoprotein as a protective factor against coronary heart disease. The Framingham Study. Am J Med 1977;62: 707-714.

3. Catapano AL, Graham I, De Backer G, Wiklund O, Chapman MJ, Drexel H, et al. 2016 ESC/EAS guidelines for the management of dyslipidaemias. Eur Heart J 2016;37:2999-3058.

4. Baseline serum cholesterol and treatment effect in the Scandinavian Simvastatin Survival Study (4S). Lancet 1995; 345: 1274-1275.

5. Baigent C, Keech A, Kearney PM, Blackwell L, Buck G, Pollici-

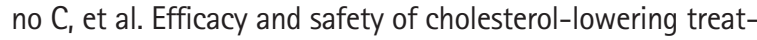
ment: prospective meta-analysis of data from 90,056 participants in 14 randomised trials of statins. Lancet 2005;366: 1267-1278.

6. Mora S, Glynn RJ, Boekholdt SM, Nordestgaard BG, Kastelein $J J$, Ridker PM. On-treatment non-high-density lipoprotein cholesterol, apolipoprotein B, triglycerides, and lipid ratios in relation to residual vascular risk after treatment with potent statin therapy: JUPITER (justification for the use of statins in prevention: an intervention trial evaluating rosuvastatin). $J$ Am Coll Cardiol 2012;59:1521-1528.

7. Olsson AG, Schwartz GG, Szarek M, Sasiela WJ, Ezekowitz MD, Ganz P, et al. High-density lipoprotein, but not lowdensity lipoprotein cholesterol levels influence short-term prognosis after acute coronary syndrome: results from the MIRACL trial. Eur Heart J 2005;26:890-896.

8. Ray KK, Cannon CP, Cairns R, Morrow DA, Ridker PM, Braunwald E. Prognostic utility of apoB/Al, total cholesterol/ HDL, non-HDL cholesterol, or hs-CRP as predictors of clinical risk in patients receiving statin therapy after acute coronary syndromes: results from PROVE IT-TIMI 22. Arterioscler Thromb Vasc Biol 2009;29:424-430.

9. Barter PJ, Caulfield M, Eriksson M, Grundy SM, Kastelein JJ, Komajda $M$, et al. Effects of torcetrapib in patients at high risk for coronary events. N Eng/J Med 2007;357:2109-2122.

10. Schwartz GG, Olsson AG, Abt M, Ballantyne CM, Barter PJ, Brumm J, et al. Effects of dalcetrapib in patients with a recent acute coronary syndrome. N Engl J Med 2012;367:2089-2099.

11. HPS3/TIMI55-REVEAL Collaborative Group, Bowman L, Hopewell JC, Chen F, Wallendszus K, Stevens W, et al. Effects of anacetrapib in patients with atherosclerotic vascular disease. N Eng/ J Med 2017;377:1217-1227.

12. Doggrell SA. What have we learnt from the clinical outcomes trials with the cetrapibs? Curr Opin Lipidol 2018;29:327-332.

13. Katakami N, Kim YS, Kawamori R, Yamasaki Y. The phosphodiesterase inhibitor cilostazol induces regression of carotid atherosclerosis in subjects with type 2 diabetes mellitus: principal results of the Diabetic Atherosclerosis Prevention by Cilostazol (DAPC) study: a randomized trial. Circulation 2010;121:2584-2591.

14. Tani T, Uehara K, Sudo T, Marukawa K, Yasuda Y, Kimura Y. Cilostazol, a selective type III phosphodiesterase inhibitor, decreases triglyceride and increases HDL cholesterol levels by increasing lipoprotein lipase activity in rats. Atherosclerosis 2000;152:299-305.

15. Kwon SU, Hong KS, Kang DW, Park JM, Lee JH, Cho YJ, et al. Efficacy and safety of combination antiplatelet therapies in patients with symptomatic intracranial atherosclerotic stenosis. Stroke 2011;42:2883-2890.

16. Kim DE, Kim JY, Jeong SW, Cho YJ, Park JM, Lee JH, et al. Association between changes in lipid profiles and progression of symptomatic intracranial atherosclerotic stenosis: a prospective multicenter study. Stroke 2012;43:1824-1830.

17. Yamashita S, Matsuzawa Y. Where are we with probucol: a new life for an old drug? Atherosclerosis 2009;207:16-23.

18. Sirtori CR, Mombelli G. Viability of developing CETP inhibitors. Cardiovasc Ther 2008;26:135-146.

19. Kim BJ, Lee EJ, Kwon SU, Park JH, Kim YJ, Hong KS, et al. Prevention of cardiovascular events in Asian patients with ischaemic stroke at high risk of cerebral haemorrhage (PICASSO): a multicentre, randomised controlled trial. Lancet Neurol 2018;17:509-518.

20. Hong KS, Kim BJ, Lee JY, Kwon SU; PICASSO Investigators. Rationale and design of the Preventlon of CArdiovascular events in iSchemic Stroke patients with high risk of cerebral hemOrrhage (PICASSO) study: a randomized controlled trial. Int J Stroke 2015;10:1153-1158. 
21. Yokoi H, Daida H, Kuwabara $Y$, Nishikawa H, Takatsu F, Tomi-

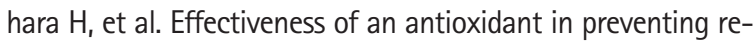
stenosis after percutaneous transluminal coronary angioplasty: the Probucol Angioplasty Restenosis Trial. J Am Coll Cardiol 1997;30:855-862.

22. Tardif JC, Cöté G, Lespérance J, Bourassa M, Lambert J, Doucet $S$, et al. Probucol and multivitamins in the prevention of restenosis after coronary angioplasty. Multivitamins and Probucol Study Group. N Engl J Med 1997;337:365-372.

23. Sawayama Y, Shimizu C, Maeda N, Tatsukawa M, Kinukawa $\mathrm{N}$, Koyanagi $\mathrm{S}$, et al. Effects of probucol and pravastatin on common carotid atherosclerosis in patients with asymptomatic hypercholesterolemia. Fukuoka Atherosclerosis Trial (FAST). J Am Coll Cardiol 2002;39:610-616.

24. Holmes MV, Asselbergs FW, Palmer TM, Drenos F, Lanktree $\mathrm{MB}$, Nelson $\mathrm{CP}$, et al. Mendelian randomization of blood lipids for coronary heart disease. Eur Heart J 2015;36:539-550.

25. Voight BF, Peloso GM, Orho-Melander M, Frikke-Schmidt $R_{1}$ Barbalic M, Jensen MK, et al. Plasma HDL cholesterol and risk of myocardial infarction: a Mendelian randomisation study. Lancet 2012;380:572-580.

26. Khera AV, Cuchel $M$, de la Llera-Moya $M$, Rodrigues $A$, Burke $M F$, Jafri $K$, et al. Cholesterol efflux capacity, high-density li- poprotein function, and atherosclerosis. N Engl J Med 2011; 364:127-135.

27. Ishigami M, Yamashita S, Sakai N, Hirano K, Arai T, Maruyama T, et al. High-density lipoproteins from probucoltreated patients have increased capacity to promote cholesterol efflux from mouse peritoneal macrophages loaded with acetylated low-density lipoproteins. Eur J Clin Invest 1997; 27:285-292.

28. Fruebis J, Gonzalez V, Silvestre M, Palinski W. Effect of probucol treatment on gene expression of VCAM-1, MCP-1, and MCSF in the aortic wall of LDL receptor-deficient rabbits during early atherogenesis. Arterioscler Thromb Vasc Biol 1997;17: 1289-1302.

29. Lau AK, Leichtweis SB, Hume P, Mashima R, Hou JY, Chaufour $X$, et al. Probucol promotes functional reendothelialization in balloon-injured rabbit aortas. Circulation 2003;107:20312036.

30. Takechi R, Galloway S, Pallebage-Gamarallage MM, Lam V, Dhaliwal SS, Mamo JC. Probucol prevents blood-brain barrier dysfunction in wild-type mice induced by saturated fat or cholesterol feeding. Clin Exp Pharmacol Physiol 2013;40:45-52.

31. Caplan LR. Lacunar infarction and small vessel disease: pathology and pathophysiology. J Stroke 2015;17:2-6. 